\title{
纳米稀土氧化物/硫酸酯化壳聚糖杂化材料的制备及其抗凝血性能研究
}

\author{
高琦宽 ${ }^{a, c}$ 王坤杰 ${ }^{b}$ 王喜存*,a \\ $\left({ }^{a}\right.$ 西北师范大学化学化工学院 兰州 730070) \\ ( $b$ 兰州理工大学石油化工学院 兰州 730050) \\ ( ${ }^{c}$ 甘肃省卫生学校 兰州 730000)
}

\begin{abstract}
摘要 利用接枝的方法制备了 4 种纳米 $\mathrm{RE}_{2} \mathrm{O}_{3}$ /硫酸酯化壳聚糖杂化材料 $\left(\mathrm{Nd}_{2} \mathrm{O}_{3}-\mathrm{TDI}-\mathrm{CS}, \mathrm{Eu}_{2} \mathrm{O}_{3}-\mathrm{TDI}-\mathrm{CS}, \mathrm{La}_{2} \mathrm{O}_{3}-\mathrm{TDI}-\mathrm{CS}\right.$, $\mathrm{Sc}_{2} \mathrm{O}_{3}$-TDI-CS, TDI=甲苯二异氰酸酯, $\mathrm{CS}$ =壳聚糖), 用红外光谱、热重分析和扫描电镜表征了产物, 结果表明壳聚糖 接枝于经过活化后的纳米氧化物表面. 抗凝血实验结果和复钙实验结果说明制备的 4 种杂化材料比纳米稀土氧化物具 有更好的抗凝血性能.
\end{abstract}

关键词 壳聚糖; 纳米稀土氧化物; 杂化材料; 抗凝血; 复䥻

\section{Study on Syntheses and Anticoagulant Action of Rare Earth Nano-oxides/TDI-CS Hybrid Materials}

\author{
Gao, Qikuan ${ }^{a, c} \quad$ Wang, Kunjie ${ }^{b}$ Wang, Xicun*,a \\ $\left({ }^{a}\right.$ College of Chemistry and Chemical Engineering, Northwest Normal University, Lanzhou 730070) \\ $\left({ }^{b}\right.$ College of Petrochemical Technology, Lanzhou University of Technology, Lanzhou 730050) \\ ( ${ }^{b}$ Gansu Health School, Lanzhou 730000)
}

\begin{abstract}
Four hybrid materials of $\mathrm{RE}_{2} \mathrm{O}_{3}$-TDI-CS (TDI = toluene 2,4-diisocyanate, $\mathrm{CS}=$ chitosan, $\mathrm{RE}=$ $\mathrm{Nd}, \mathrm{Eu}, \mathrm{La}, \mathrm{Sc}$ ) were prepared by the method of graft. The materials were characterized by infrared spectroscopy, thermogravimetric and scanning electron microscopy, and results showed that the CS was grafted on the surface of rare earth nano-oxides which was modified by TDI. The results of the experiment of anticoagulant and recalcification time showed that the materials have better anticoagulant action than that of $\mathrm{RE}_{2} \mathrm{O}_{3}$.
\end{abstract}

Keywords chitosan (CS); rare earth nano-oxide; hybrid material; anticoagulant; recalcification

壳聚糖 $(\mathrm{CS})$ 是一类重要的天然生物高分子材料, 是 甲壳素的衍生物, 是地球上仅次于纤维素的第二大可再 生资源, 属含氨基的均态直链多糖, 化学名为聚(1,4)-2氨基-2-脱氧 $-\beta-D$ 葡聚糖, 是天然多糖中唯一的碱性多 糖, 也是少数具有荷电性的天然产物之一, 具有许多独 特的物理、化学性质和生物功能 ${ }^{[1,2]}$. 由于 CS 分子内存 在游离的氨基、羟基, 因而可以进一步改性, 其衍生物
不但溶解性会大大增加、生物活性显著增强，且其衍生 物具有良好的抗凝血性能 ${ }^{[3]}$. 近年来稀土化合物的抗凝 血作用受到人们的关注 ${ }^{[4]}$, 已得到广泛的研究和应用, 其特点是起效快、持续时间长，而且配合物稳定. 但在 临床应用方面由于稀土离子的毒性和累积问题而受到 一定限制 ${ }^{[5]}$. 因此，合成高效、低毒稀土抗凝血药物，在 医学、生物学和化学等学科领域有着重要的理论意义.

*E-mail: wangluooda@163.com

Received March 23, 2011; revised July 7, 2011; accepted September 14, 2011.

Project supported by the National Natural Science Foundation of China (No. 21062017) and Natural Science Foundation of Gansu Province (No. 1112RJZA006), Gansu Key Laboratory of Polymer Materials and Lanzhou University of Technology for Ph.D.

国家自然科学基金(No. 21062017)、甘肃省自然科学基金(No. 1112RJZA006)和甘肃省高分子材料重点实验室和兰州理工大学博士科研启动资助项目. 
本课题组曾系统研究过稀土华发灵配合物、稀土华发灵 水杨酸三元配合物、稀土半胱氨酸枸侬酸三元配合物的 抗凝血性能 ${ }^{[6 ~ 8]}$. 结果表明, 配合物的抗凝血性能均比 配体好; 二元配合物的抗凝血性能比三元配合物好, 但 三元配合物的水溶性比二元配合物好. 为了得到抗凝血. 性能和水溶性更好的配合物, 本课题组设计和制备了 4 种纳米 $\mathrm{RE}_{2} \mathrm{O}_{3}$ /壳聚糖杂化材料, 对其进行了红外光谱、 热重分析和扫描电镜等分析表征, 并研究了其抗凝血性 质. 制备的 4 种杂化材料的抗凝血性能比上述配合物的 大大提高(相同条件下，配合物的抗凝血时间不超过 15 $\min$, 杂化材料在 $20 \mathrm{~min}$ 以上), 水溶性也比配合物好(配 合物溶液要用有机溶剂或滴加表面活性剂配制, 杂化材 料溶液直接用水配制).

\section{1 实验部分}

\section{1 试剂及材料}

$\mathrm{RE}_{2} \mathrm{O}_{3} 、 \mathrm{TDI}$ (甲苯二异氧酸酯)、硬脂酸、丙酮、二 丁基二月桂酸锡、三甲基铵三氧化硫、四氢呋喃、壳聚 糖、甲醇、过硫酸铵. 以上试剂均为分析纯.

\section{2 甲苯二异氭酸酯对纳米稀土氧化物表面有机改性}

纳米稀土氧化物用硬脂酸法制得 ${ }^{[9]}$. 将纳米稀土氧 化物在真空烘箱中 $120{ }^{\circ} \mathrm{C}$ 烘 $7 \sim 8 \mathrm{~h}$. 称取预处理过的纳 米稀土氧化物 $5 \mathrm{~g}$, 甲苯-2,4-二异氞酸酯 $5 \mathrm{~g}$ 和 $100 \mathrm{~mL}$ 无水丙酮加入三口烧瓶中, 在氮气保护下室温超声处理 $2 \mathrm{~h}$ 后, 加入适量的二丁基二月桂酸锡, $80{ }^{\circ} \mathrm{C}$ 超声反应 2 h. 经离心, 洗涤, 真空干燥得固体, 样品标记为 $\mathrm{RE}_{2} \mathrm{O}_{3}$-TDI.

\section{3 硫酸酯化壳聚糖的制备}

将壳聚糖经冷冻干燥处理后, 分散于水中, 加入 $\mathrm{Na}_{2} \mathrm{CO}_{3}$ 和 $\mathrm{Me}_{3} \mathrm{~N}-\mathrm{SO}_{3}$, 在 $\mathrm{pH}=9.5$ 下, $65{ }^{\circ} \mathrm{C}$ 反应 $12 \mathrm{~h}$ 至 形成透明的粘性溶液, 冷却后依次用蒸馏水、离子交换 树脂的蒸馏水、 $\mathrm{NaOH}$ 溶液和蒸馏水透析, 再冷冻干燥 可得硫酸酯化壳聚糖.

\section{4 硫酸酯化壳聚糖/纳米稀土氧化物杂化材料的制备}

称取 $1 \mathrm{~g} \mathrm{RE}_{2} \mathrm{O}_{3}$-TDI 加入到 $50 \mathrm{~mL}$ THF 中, 超声分 散 $1 \mathrm{~h}$ 后, 加入 $2.5 \mathrm{~g}$ 硫酸酯化壳聚糖, 再加入 $5 \mathrm{~mL}$ 甲 醇, 氮气保护下室温搅拌 $2 \mathrm{~h}$. 经离心, 洗涤, 真空干燥 得固体, 再加入 $50 \mathrm{~mL}$ THF 及 $1 \mathrm{~g}$ 硫酸酯化壳聚糖和 10 $\mathrm{mL}$ 浓度为 $25 \%$ 的过硫酸铵水溶液, 室温反应 $4 \mathrm{~h}$ 后, 过 滤, 洗涤, $50{ }^{\circ} \mathrm{C}$ 真空干燥 $24 \mathrm{~h}$ 后得固体. 产品分别标记 为 $\mathrm{RE}_{2} \mathrm{O}_{3}$-TDI-CS.

\section{5 分析与表征}

样品及中间产物的红外光谱是在室温下用 Nicolet
NEXLS 670 傅里叶红外转变分光计(FT-IR)进行测试的, 采用 $\mathrm{KBr}$ 压片法, 波段范围从 400 到 $4000 \mathrm{~cm}^{-1}$; 热失重 分析(TGA)表征采用的仪器是 Perkin-Elmer TGA-7 型热 失重仪(Perkin-Elmer Corporation, USA), 升温速度为 10 ${ }^{\circ} \mathrm{C} / \mathrm{min}$, 在 $\mathrm{N}_{2}$ 保护下升温到 $800{ }^{\circ} \mathrm{C}$; 产品的表面形貌 的表征采用的仪器是扫描电子显微镜(XL-20, 飞利浦公 司, 荷兰), 操作电压为 $25 \mathrm{kV}$.

\section{6 抗凝血试验}

\subsection{1 全血凝血试验}

取洁净的试管分为两组, 一组为空白, 一组为样品, 样品试管加入一定量的待测抗凝剂 $(0.01 \mathrm{~g})$, 每组 3 个平 行样, 试管经 $37{ }^{\circ} \mathrm{C}$ 恒温后, 沿管壁加入 $1 \mathrm{~mL}$ 兔血于 试管中, $37{ }^{\circ} \mathrm{C}$ 恒温, $2 \mathrm{~min}$ 后, 每隔 $10 \mathrm{~s}$ 轻轻的倾斜试 管, 倾斜动作要轻, 角度约 $30^{\circ}$, 直至血液不再流动, 记 录该时间为全血凝血. 时间 ${ }^{[10]}$.

\subsection{2 复钙试验}

将待测样品放入质量分数 0.009 的 $\mathrm{NaCl}$ 溶液中浸 泡 $24 \mathrm{~h}$, 取两组洁净的试管(每组 3 个) $37{ }^{\circ} \mathrm{C}$ 恒温后, 将 浸泡过的样品转移到一组洁净的试管中(每支 $0.01 \mathrm{~g}$ ), 另一组为空白, 分别加入 $0.3 \mathrm{~mL}$ 兔血, $0.025 \mathrm{~mol} \cdot \mathrm{L}^{-1}$ 的 $\mathrm{CaCl}_{2} 0.3 \mathrm{~mL}, 37^{\circ} \mathrm{C}$ 恒温, 将一根不锈钢小钩伸入溶液 中均匀缓慢地摚动, 并检查是否有纤维蛋白的形成. 记 录小钩上刚开始出现丝状物的时间，此时间即是复铻化 凝血时间 ${ }^{[11]}$.

\section{2 结果与讨论}

\section{$2.1 \mathrm{IR}$ 分析}

图 1 为 $\mathrm{Nd}_{2} \mathrm{O}_{3}$-TDI-CS 及中间产物的红外光谱图. 根据文献[12,13]认为: $1624 \mathrm{~cm}^{-1}$ 和 $3435 \mathrm{~cm}^{-1}$ 的强振动 吸收峰表明了 $\mathrm{Nd}_{2} \mathrm{O}_{3}$ 表面羟基的存在. 与纳米 $\mathrm{Nd}_{2} \mathrm{O}_{3}$ 相 比, $\mathrm{Nd}_{2} \mathrm{O}_{3}$-TDI 在 $1650 \mathrm{~cm}^{-1}$ 和 $1541 \mathrm{~cm}^{-1}$ 处出现了氨基 甲酸酯的吸收峰 ${ }^{[14]} ; 1605 \mathrm{~cm}^{-1}$ 处存在苯环的吸收峰; $2915 \mathrm{~cm}^{-1}$ 和 $2857 \mathrm{~cm}^{-1}$ 处出现了 TDI 的甲基的 C-H 不 对称伸缩振动和对称伸缩振动吸收峰, 这说明 TDI 和纳 米 $\mathrm{Nd}_{2} \mathrm{O}_{3}$ 表面的羟基发生了反应，TDI 已经接枝到纳米 $\mathrm{Nd}_{2} \mathrm{O}_{3}$ 表面. 然而在 $2277 \mathrm{~cm}^{-1}$ 处仍然存在着一 $\mathrm{NCO}$ 的 特征吸收峰, 可以断定 TDI 的两个一NCO 中反应活性 较弱的一个得以保留 ${ }^{[15]}$. 而在 $\mathrm{Nd}_{2} \mathrm{O}_{3}$-TDI-CS 的 IR 图中, $2277 \mathrm{~cm}^{-1}$ 处的一 NCO 的特征吸收峰消失, 说明-NCO 和壳聚糖反应, 使得硫酸酯化壳聚糖接枝在 $\mathrm{Nd}_{2} \mathrm{O}_{3}$-TDI 的表面. 在 $\mathrm{Nd}_{2} \mathrm{O}_{3}$-TDI-CS 的 IR 图中, 还出现了 1726 $\mathrm{cm}^{-1}$ 处的酯羰基 $(\mathrm{C}=\mathrm{O})$ 的伸缩振动吸收峰, 同时, 在 $3432 \mathrm{~cm}^{-1}$ 处出现较宽、较强的羟基吸收峰, 在 590 $\mathrm{cm}^{-1}$ 处出现了 $\mathrm{Nd}-\mathrm{O}$ 的特征吸收峰. 这些均说明壳聚 
糖成功地接枝在了改性的 $\mathrm{Nd}_{2} \mathrm{O}_{3}$ 表面. 其它三种杂化材 料的 IR 图谱和 $\mathrm{Nd}_{2} \mathrm{O}_{3}$-TDI-CS 的较为相似.

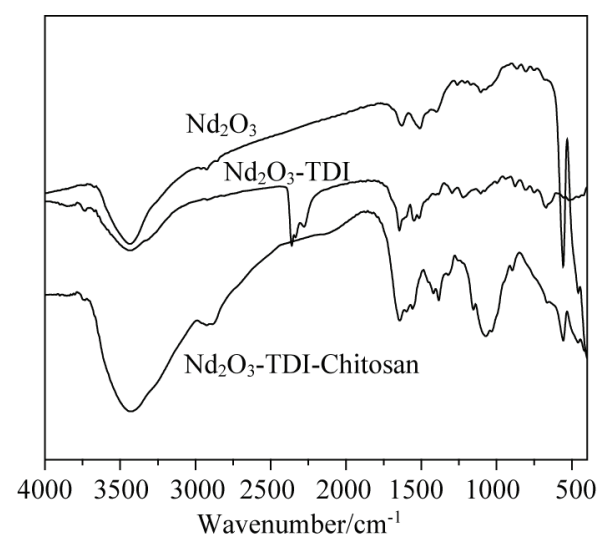

图 1 纳米 $\mathrm{Nd}_{2} \mathrm{O}_{3}$ 改性前后的 IR 图谱

Figure 1 The IR spectra of nano- $\mathrm{Nd}_{2} \mathrm{O}_{3}$ before and after the grafting

\subsection{TG 分析}

图 2(a $\sim \mathrm{d})$ 分别为 $\mathrm{Nd}_{2} \mathrm{O}_{3}$-TDI-CS, $\mathrm{Eu}_{2} \mathrm{O}_{3}$-TDI-CS, $\mathrm{La}_{2} \mathrm{O}_{3}$-TDI-CS, $\mathrm{Sc}_{2} \mathrm{O}_{3}$-TDI-CS 及中间产物的 TG 曲线. 从图中可以看出, $200{ }^{\circ} \mathrm{C}$ 以下, $\mathrm{RE}_{2} \mathrm{O}_{3}$ 的失重率约为 $1.22 \%$ 左右，归属于纳米粒子表面羟基吸附水的脱去,
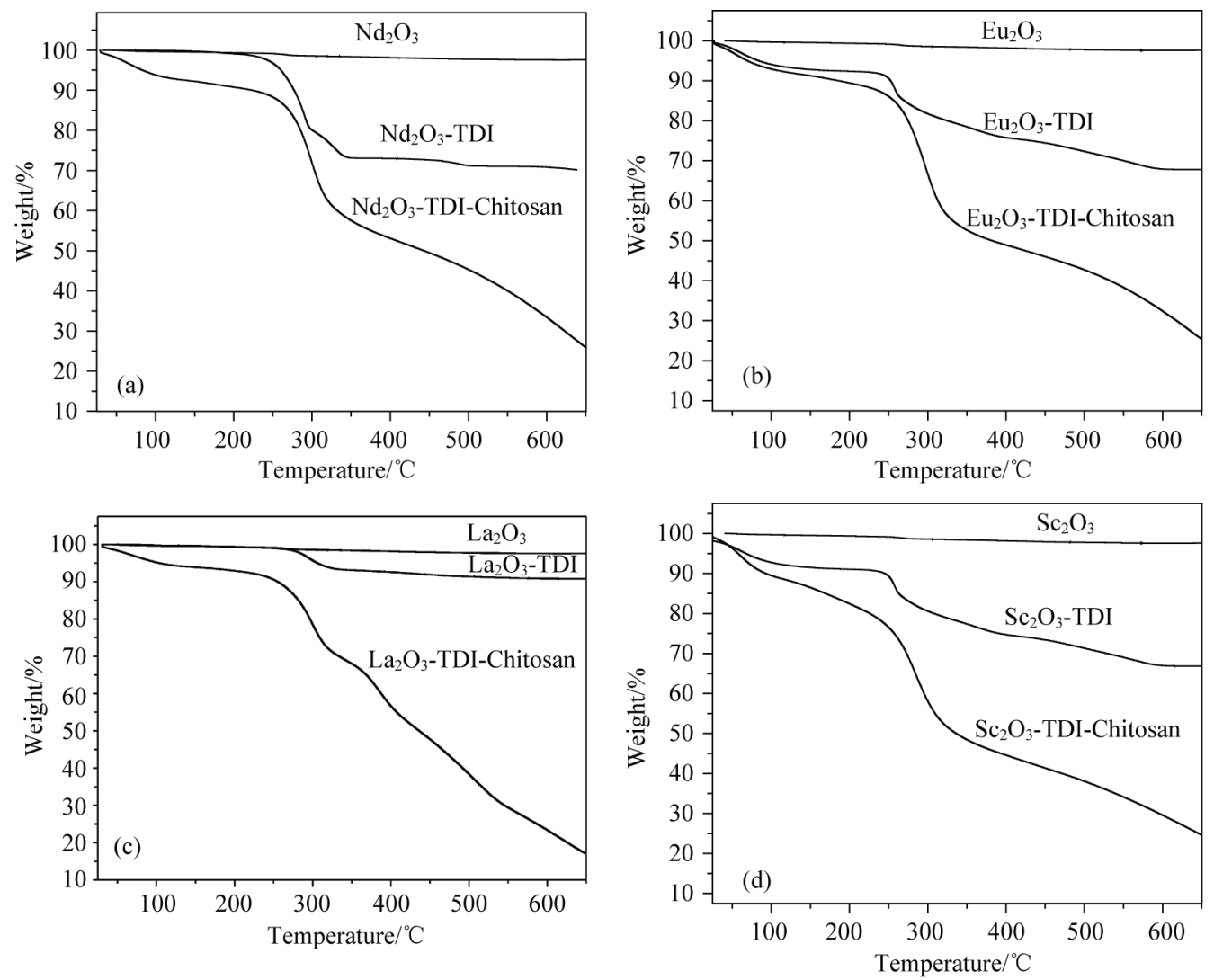

图 2 纳米稀土氧化物改性前后的 TG 曲线

Figure 2 The weight loss curves for the nano- $\mathrm{RE}_{2} \mathrm{O}_{3}$ before and after the grafting $\mathrm{a}=\mathrm{Nd}_{2} \mathrm{O}_{3}, \mathrm{~b}=\mathrm{Eu}_{2} \mathrm{O}_{3}, \mathrm{c}=\mathrm{La}_{2} \mathrm{O}_{3}, \mathrm{~d}=\mathrm{Sc}_{2} \mathrm{O}_{3}$ $51.2 \mathrm{wt} \%, 52.3 \mathrm{wt} \%, 64.2 \mathrm{wt} \%$ 和 $49.8 \mathrm{wt} \%$.

\subsection{SEM 分析} 将 $\mathrm{RE}_{2} \mathrm{O}_{3}$ 粒子完全包覆.

\section{4 反应原理}

温度在 $200 \sim 600{ }^{\circ} \mathrm{C}$ 之间; 而经过甲苯-2,4-二异氧酸酯 改性后, $\mathrm{RE}_{2} \mathrm{O}_{3}$ - TDI 在 $160 \sim 600{ }^{\circ} \mathrm{C}$ 的失重主要是 $\mathrm{RE}_{2} \mathrm{O}_{3}$-TDI 表面的 TDI 的分解，热失重分析表明纳米 $\mathrm{RE}_{2} \mathrm{O}_{3}$ 表面分别包覆了 TDI. 由此进一步说明 $\mathrm{RE}_{2} \mathrm{O}_{3}$ 的 表面有机改性是成功的. 而 $\mathrm{RE}_{2} \mathrm{O}_{3}$-TDI-CS 的 TG 曲线在 $160{ }^{\circ} \mathrm{C}$ 以后出现明显的失重台阶，这些失重可以认为是 由接枝到 $\mathrm{RE}_{2} \mathrm{O}_{3}$ 表面的硫酸酯化壳聚糖分解所引起的. 根据接枝上的 CS 与加入的无机纳米粒子质量之比可计 算出 $\mathrm{CS}$ 的接枝率 ${ }^{[16]}$, 由失重结果可知, 接枝率分别为

图 3(a $\sim d)$ 分别为 $\mathrm{Nd}_{2} \mathrm{O}_{3}$-TDI-CS, $\mathrm{Eu}_{2} \mathrm{O}_{3}$-TDI-CS, $\mathrm{La}_{2} \mathrm{O}_{3}$-TDI-CS, $\mathrm{Sc}_{2} \mathrm{O}_{3}$-TDI-CS 的扫描电镜图. 从图中可 以看到, $\mathrm{CS}-\mathrm{RE}_{2} \mathrm{O}_{3}$ 均为纳米层状结构的固体, 长度在 $50 \sim 400 \mathrm{~nm}$ 不等，而看到的 $\mathrm{RE}_{2} \mathrm{O}_{3}$ 粒子很少，这表明在 $\mathrm{RE}_{2} \mathrm{O}_{3}$ 表面成功地发生了接枝反应, 硫酸酯化壳聚糖已

$\mathrm{RE}_{2} \mathrm{O}_{3}$-TDI-CS 的制备过程如图 4 所示. 杂化材料 的制备, 是利用 TDI 分子中二个一 $\mathrm{NCO}$ 基团可分别桥 


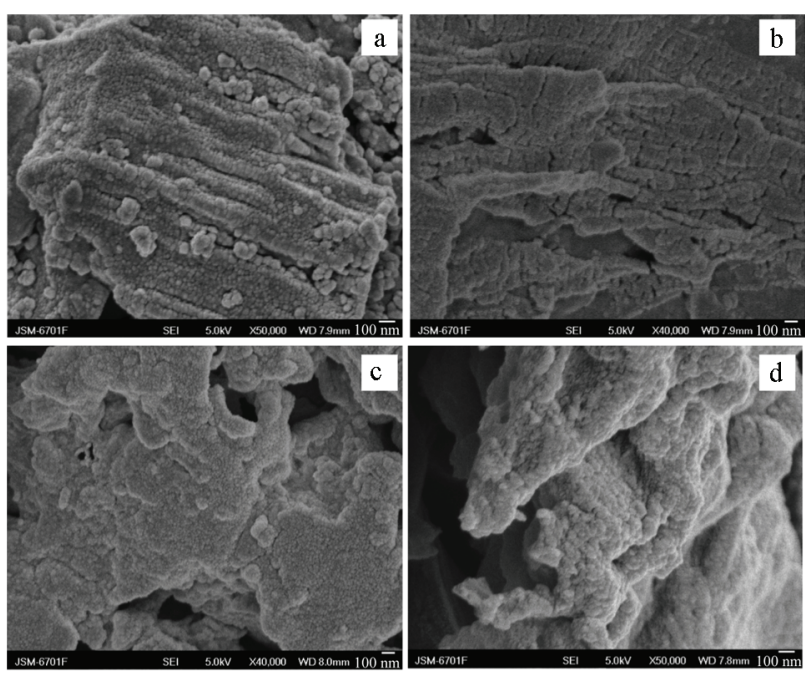

图 $3 \mathrm{RE}_{2} \mathrm{O}_{3}$-TDI-CS 的扫描电镜图

Figure 3 The SEM of $\mathrm{RE}_{2} \mathrm{O}_{3}$-TDI-CS $\mathrm{a}=\mathrm{Nd}_{2} \mathrm{O}_{3}, \mathrm{~b}=\mathrm{Eu}_{2} \mathrm{O}_{3}, \mathrm{c}=\mathrm{La}_{2} \mathrm{O}_{3}, \mathrm{~d}=\mathrm{Sc}_{2} \mathrm{O}_{3}$
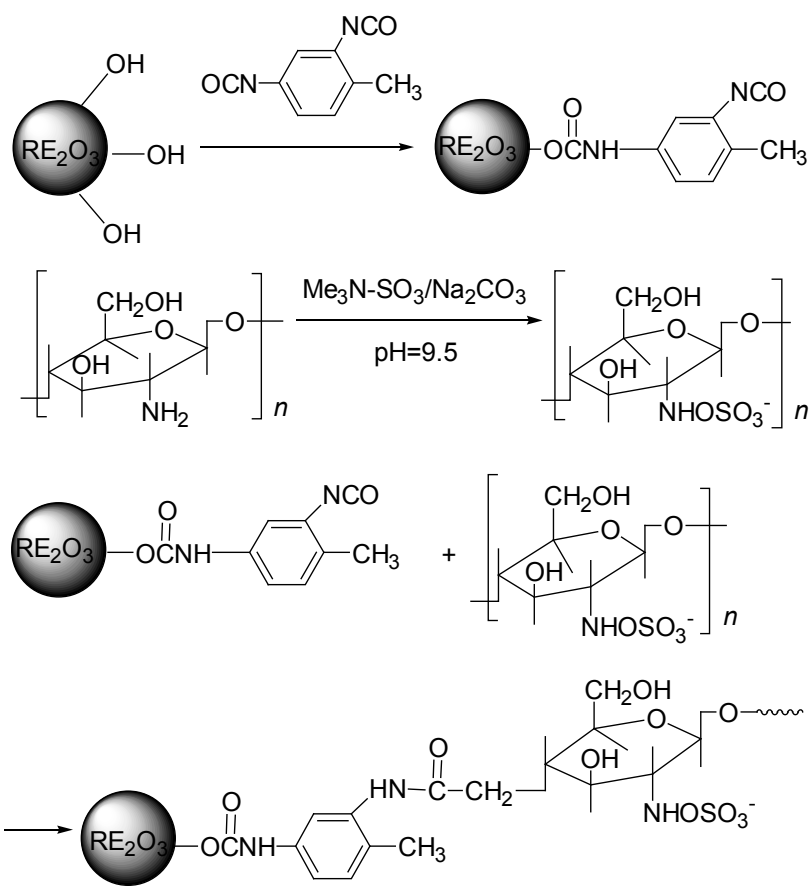

图 $4 \mathrm{RE}_{2} \mathrm{O}_{3}$-TDI-CS 的制备过程

Figure 4 The process of preparation of $\mathrm{RE}_{2} \mathrm{O}_{3}$-TDI-CS

联 $\mathrm{RE}_{2} \mathrm{O}_{3}$ 纳米粒子和 $\mathrm{CS}$ 来实现的. 即: 将表面具有羟 基的 $\mathrm{RE}_{2} \mathrm{O}_{3}$ 纳米粒子与 $\mathrm{TDI}$ 反应，纳米粒子表面的羟基 与 TDI 上的一个一 $\mathrm{NCO}$ 基团发生反应生成 $\mathrm{RE}_{2} \mathrm{O}_{3}$-TDI 复合物，该复合物中的另一个一 $\mathrm{NCO}$ 基团和硫酸酯化 壳聚糖分子中的羟基反应, 使硫酸酯化壳聚糖接枝于纳 米粒子表面.

\section{5 抗凝血试验}

\subsection{1 全血凝血时间(CT min)}

图 5 为纳米 $\mathrm{RE}_{2} \mathrm{O}_{3}, \mathrm{RE}_{2} \mathrm{O}_{3}-\mathrm{TDI}, \mathrm{RE}_{2} \mathrm{O}_{3}$-TDI-CS 和对
照组的全血凝血时间 $\left(1=\right.$ nano- $\mathrm{RE}_{2} \mathrm{O}_{3}, 2=\mathrm{RE}_{2} \mathrm{O}_{3}$-TDI, $3=\mathrm{RE}_{2} \mathrm{O}_{3}$-TDI-CS), 从图中可以看出, 与对照组相比, 纳米稀土氧化物/硫酸酯化壳聚糖杂化材料具有很好的 抗凝血作用, 并且可以看出, 杂化材料的抗凝效果明显 好于纳米 $\mathrm{RE}_{2} \mathrm{O}_{3}$ 和 $\mathrm{RE}_{2} \mathrm{O}_{3}$-TDI. 4 种杂化材料中, 销、锞 和锌杂化材料的抗凝血时间几乎相同，而钕杂化材料的 抗凝血时间比其它三种材料的时间略长. 由于杂化材料 的抗凝血性质主要来自壳聚糖, 而稀土离子的化学性质 较为相似, 故 4 种杂化材料的抗凝血时间长短差别不大. 利用硫酸酯化壳聚糖制备的具有抗凝血作用的纳米稀 土氧化物/硫酸酯化壳聚糖杂化材料, 为本课题组继续 研究抗凝配合物的配体选择和设计提供了一定的实验 基础.

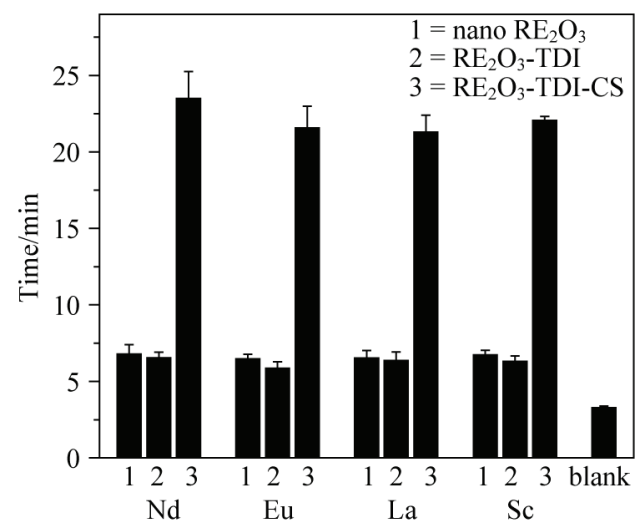

图 5 纳米 $\mathrm{RE}_{2} \mathrm{O}_{3}, \mathrm{RE}_{2} \mathrm{O}_{3}-\mathrm{TDI}, \mathrm{RE}_{2} \mathrm{O}_{3}-\mathrm{TDI}-\mathrm{CS}$ 和对照组的全血. 凝血时间

Figure 5 The whole blood coagulation time of nano $\mathrm{RE}_{2} \mathrm{O}_{3}$, $\mathrm{RE}_{2} \mathrm{O}_{3}$-TDI, $\mathrm{RE}_{2} \mathrm{O}_{3}$-TDI-CS and blank

\subsection{2 复钻时间 (RT)}

图 6 为纳米 $\mathrm{RE}_{2} \mathrm{O}_{3}, \mathrm{RE}_{2} \mathrm{O}_{3}-\mathrm{TDI}, \mathrm{RE}_{2} \mathrm{O}_{3}-\mathrm{TDI}-\mathrm{CS}$ 和对 照组的复铻时间. 从图中也可以看出, 杂化材料 $\mathrm{RE}_{2} \mathrm{O}_{3}$ -

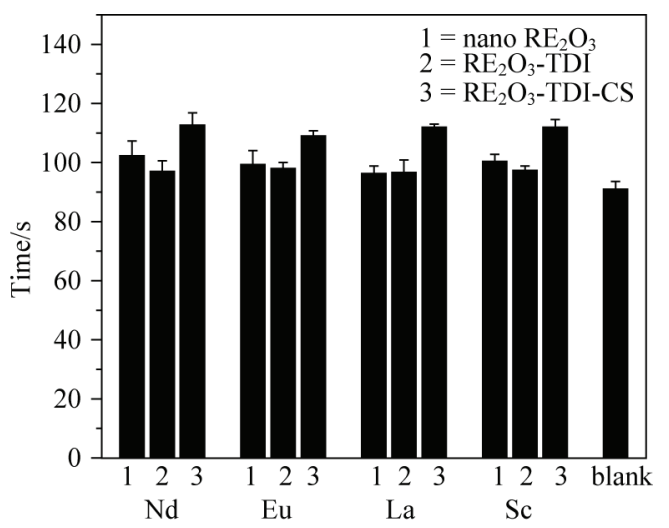

图 6 纳米 $\mathrm{RE}_{2} \mathrm{O}_{3}, \mathrm{RE}_{2} \mathrm{O}_{3}-\mathrm{TDI}, \mathrm{RE}_{2} \mathrm{O}_{3}-\mathrm{TDI}-\mathrm{CS}$ 和对照组的复钘 时间

Figure 6 The recalcification time of nano- $\mathrm{RE}_{2} \mathrm{O}_{3}, \mathrm{RE}_{2} \mathrm{O}_{3}$-TDI, $\mathrm{RE}_{2} \mathrm{O}_{3}$-TDI-CS and blank 
TDI-CS 相对于对照组样品来说, 明显延长了复钙的时 间，同时 4 种杂化材料的复钻时间比相应的纳米 $\mathrm{RE}_{2} \mathrm{O}_{3}$ 和 $\mathrm{RE}_{2} \mathrm{O}_{3}$-TDI 的时间长, 说明杂化材料抗凝效果好于纳 米 $\mathrm{RE}_{2} \mathrm{O}_{3}$ 和 $\mathrm{RE}_{2} \mathrm{O}_{3}$-TDI, 说明其具有良好的抗凝血作用. 4 种杂化材料的复钙时间几乎没有差别, 这与稀土离子 性质相似有关.

\section{3 结论}

利用 TDI(甲苯-2,4-二异氰(酸酯)活化纳米稀土氧化 物和接枝的方法制备了稀土元素钕、铕、镧和钪的纳米 氧化物/硫酸酯化壳聚糖杂化材料, 抗凝血实验及复钙 实验说明纳米稀土氧化物/硫酸酯化壳聚糖杂化材料具 有良好的抗凝血性能. 和本课题组所合成并报道的稀土 华发灵配合物、稀土华发灵水杨酸三元配合物、稀土半 胱氨酸枸椽酸三元配合物的抗凝血性能比较, 本论文所 报道的杂化材料具有更好的抗凝血作用和较好的水溶 性.

\section{References}

1 Muzzarelli, R. A. Chitin, Pergamon Press, New York, 1997, pp. $1 \sim 37$.

2 Roberts, G. F. Chitin Chemistry, Mac Millian Press, Houndmills, 1992, pp. 1 $\sim 50$.

3 Jiang, Z.-J.; Wang, Z.-Y.; Hu, X.-Q. Chem. Bioeng. 2008, 12, 57 (in Chinese).

(蒋珍菊, 王周玉, 胡星琪, 化学与生物工程, 2008, 12 , 57.)

4 Funakoshi, T.; Furushima, K.; Shimada, H. Biochem. Int 1992, 28, 113.
5 Novikova, N. V.; Lobanova, E. G.; Lebedeva, E. N.; Men'kov, A. A.; Galaktionova, O. V. Farmakol. Toksikol. (Moscow) 1986, 49, 32.

6 Wang, K.-J.; Luan, N.-N.; Song, Y.-M. Acta Chim. Sinica 2009, 67, 1042 (in Chinese).

(王坤杰, 奕尼娜, 宋玉民, 化学学报, 2009, 67, 1042.)

7 Dong, Y.-L.; Luan, N.-N.; Wang, H.-Y.; Song, Y.-M. Acta Chim. Sinica 2008, 66, 1497 (in Chinese).

(董银龙, 森尼娜, 王海燕，宋玉民，化学学报， 2008，66, 1497.)

8 Song, Y.-M.; Dong, Y.-L.; Fu, Y.-L.; Yao, K.-L. Acta Chim. Sinica 2007, 65, 678 (in Chinese).

(宋玉民, 董银龙, 付云龙, 姚卡玲, 化学学报, 2007, 65, 678.)

9 Wang, X.; Lu, L.-D. Chinese J. Inorg. Chem. 2000, 16, 213 (in Chinese).

(汪信，陆路德，无机化学学报, 2000, 16, 213.)

10 Liu, P.; Chen, Y.-S.; Zhang, L.-H. J. Funct. Polym. 2004, 17, 35 (in Chinese).

(刘鹏，陈亚莳，张丽惠，功能高分子学报， 2004, 17, 35.)

11 Shi, H.-T.; Mo, X.-M.; He, C.-L.; Guo, K.; Liu, S.-H. J. Clin. Rehabilitative Tissue Eng. Res. 2008, 12, 10 (in Chinese).

(石海涛，莫秀梅，何创龙，过凯，刘生和，中国组织工程 研究与临床康复, 2008, 12, 10.)

12 Nag, M.; Basak, P.; Manorama, S. V. Mater. Res. Bull. 2007, 42,1691 .

13 Watson, S.; Beydoun, D.; Scott, J.; Amal, R. J. Nanopart. Res. 2004, 6, 193.

14 Li, H. R.; Lin, J.; Zhang, H. J.; Fu, L. S.; Meng, Q. G.; Wang, S. B. Chem. Mater. 2002, 14, 3651.

15 Lei, X. P.; Su, Z. X. Polym. Adv. Technol. 2007, 18, 472.

16 Wang, Q.; Ni, J.-Z. Chem. J. Chin. Univ. 2004, 25, 1783 (in Chinese).

(王群，倪嘉绩，高等学校化学学报, 2004, 25, 1783.)

(A1103231 Cheng, B.) 